\title{
Unternehmen oder Unvernehmen? - Über die Krise des Kreativsubjekts und darüber hinaus
}

\author{
I. Dzudzek \\ Institut für Humangeographie, Goethe-Universität, Frankfurt am Main, Germany \\ Correspondence to: I. Dzudzek (dzudzek@em.uni-frankfurt.de)
}

Received: 31 July 2012 - Revised: 7 December 2012 - Accepted: 13 December 2012 - Published: 7 October 2013

Zusammenfassung. Im Neoliberalismus sind die politischen Handlungsspielräume für das zu Selbstführung und -verwertung verdammte Kreativsubjekt eng geworden und auch im Unternehmen Stadt werden politische Prozesse zunehmend Marktlogiken und ,-zwängen“ untergeordnet. Am Beispiel der Auseinandersetzungen über die Planung eines KulturCampus in Frankfurt am Main und mit Hilfe neuerer Theorien des Politischen untersucht dieser Artikel aktuelle Formen des Unvernehmens gegen hegemoniale Formen unternehmerischer Politik und lotet neue Möglichkeiten politischer Subjektivitäten in der kreativen Stadt aus, wie sie derzeit u.a. im Kontext der Recht-auf-Stadt-Bewegung und in den performance studies erprobt werden. Dabei wird der Frage nachgegangen, inwiefern diese neuen Formen des Widerstandes in der Lage sind, die marktlogischen, postdemokratischen Regeln von Politik selbst zum Thema, neue Subjektpositionen artikulierbar und Stadt politisch wieder verhandelbar zu machen.

\section{Problemaufriss}

Das kreative Selbst ist in den vergangenen Jahren zu einer Symbolfigur krisenhafter Subjektivität geworden. Die Kritik an fordistischen Arbeitsverhältnissen und die mit ihr verbundenen Forderungen nach Freiheit, Selbstverwirklichung und -bestimmung stellen im flexiblen Kapitalismus zunehmend eine Systemressource dar. Der „,neue Geist des Kapitalismus" weiß - folgt man der These von Boltanski und Chiapello (2006) - sogar noch Kritik und kulturelle Differenz zu verwerten. Das kreative, künstlerische und schöpferische Selbst transformiert sich in eine_n Arbeitskraftunternehmer_in (Pongratz und Voß, 2003), der/die genötigt ist, sich selbst als Marke zu inszenieren, um durch die totale Selbstkapitalisierung und -ausbeutung das eigene Überleben in prekären Verhältnissen zu sichern (Loacker, 2010; Manske, 2008; McRobbie, 2009; von Osten und von Bismarck, 2003; Reckwitz, 2008). Der unlösbare Widerspruch zwischen dem Streben nach Selbstbestimmung und -verwirklichung und dem Zwang zur Selbstverwertung stürzt das kreative Selbst nicht selten in eine persönliche Krise, die sich häufig in einem zynischen Selbstverhältnis oder einer Depression zeigt (vgl. hierzu Ehrenberg, 2008; Menke und Rebentisch, 2010). Von dieser Krise des kreativen Subjekts zeugt nicht nur eine große Anzahl an Publikationen wissenschaftlicher wie populärer Art, sondern vor allem der prekäre Alltag und das schwierige Selbstverhältnis einer immer größer werdenden Gruppe an schöpferisch Tätigen.

Die genannten Analysen des Kreativsubjekts argumentieren vor einem poststrukturalistischen, oft gouvernementalitätstheoretischen Hintergrund nicht selten fatalistisch: Die gegebenen Verhältnisse im flexiblen Kapitalismus führten zu einem Zwang zur Selbstverwertung aus dem es kein Entrinnen gäbe. Dies ist insofern verwunderlich, als dass es doch gerade das Verdienst poststrukturalistischer und auch gouvernementalitätstheoretischer Ansätze ist, über den Strukturalismus hinaus zu gehen, in dem sie auch die produktive Seite von Subjektivität betonen (in der kritischen Humangeographie siehe z.B. Gibson, 2001; Gregson und Rose, 2000; McDowell, 2008; Strüver in diesem Band, 2011a, b; Valentine, 2007). Jedoch bleiben häufig in konkreten empirischen Analysen kreativer Selbste ihre widersprüchlichen Subjektivierungsformen, ihre Umkämpftheit in der kreativen Stadt und ihre oszillierende Ambivalenz in den Praktiken des Alltags ebenso unterbelichtet wie konkrete Widerstandspraktiken und gegenhegemoniale Artikulationsformen. 
Der vorliegende Beitrag nimmt die produktiven Seiten von Subjektivität in den Blick und vertritt die These, dass der fatalistischen Diagnose des vollkommen ökonomisierten und kulturalisierten Selbstes nur mit einer Repolitisierung begegnet werden kann. Repolitisierung meint dabei nicht den Rückgriff auf große politische Kategorien der Moderne oder auf klassisch linke Theorien sozialer Bewegungen. Eine Repolitisierung erfolgt im weiteren Gang der Argumentation vielmehr in einer theoretischen und einer empirischen Suchbewegung: Theoretisch geschieht sie im Rückgriff auf poststrukturalistische Theorien des Politischen und politischer Subjektivität insbesondere in Anlehnung an Butler (2008), Marchart (2010), Rancière (2004, 2007) und Swyngedouw (2005, 2011). Das Politische in den Aushandlungsprozessen der kreativen Stadt stärker in den Blick zu nehmen bedeutet, die Kategorie des widerständigen Subjekts ins Spiel zu bringen. Hierbei spielen gegenhegemoniale Strategien der Selbst- und Raumproduktion eine zentrale Rolle. Empirisch geschieht dies entlang eines Fallbeispiels, anhand dessen die Konflikte und Aushandlungsprozesse über den Bau eines „kreativen Leuchtturmprojekts“, dem sogenannten KulturCampus in Frankfurt am Main, analysiert werden. Dabei steht nicht die Krise des kreativen Subjekts in seiner gegenwärtigen Arbeitssituation im Fokus, sondern seine (Re-)Politisierung im Rahmen der Planung des KulturCampus als neuem Stadtquartier, das kreative Arbeit beherbergen wird. Hierbei geht es vor allem darum, den performativen Akten und gegenhegemonialen Formen der Artikulation und des Unvernehmens aus dem Kontext der Recht-aufStadt-Bewegung nachzuspüren, die versuchen, die durch den postpolitischen Konsens einer neoliberalen Stadtentwicklung verunmöglichten Subjektpositionen wieder in der urbanen Arena verhandelbar, performativ erlebbar und anrufbar zu machen.

Dazu wird in vier Schritten verfahren: Zunächst wird einführend das krisenhafte Kreativsubjekt im postpolitischen Konsens der unternehmerischen kreativen Stadt verortet, anschließend wird kurz in die Theorien des Politischen und performativer politischer Subjektivität eingeführt. Sodann wird anhand des Fallbeispiels nach neuen Formen des Unvernehmens und der Artikulation politischer Subjektivität gefragt. Abschließend werden noch einmal kritisch Möglichkeiten und Grenzen der Repolitisierung in theoretischer und praktischer Hinsicht diskutiert und in den Kontext der Recht-aufStadt-Bewegung gestellt.

\section{Kontextualisierung des Kreativsubjekts im postpolitischen Konsens der unternehmerischen Kreativstadt}

Das Problem der Diagnose des Kreativsubjekts als eines zu Selbstausbeutung und -verwertung verdammten Kreativunternehmers ist eng mit der gegenwärtig hegemonialen wettbewerbsorientierten Stadtpolitik (Mattissek, 2008, für Frank- furt Schipper, 2012) verwoben, die unternehmerische Subjektivität soweit naturalisiert und habitualisiert, dass sie als Sachzwang erscheint und nicht mehr hinterfragbar ist. Die zunehmende Hegemonialisierung des Diskurses der neoliberalen Kreativstadt (Peck, 2005, 2012) zeigt sich vor allem darin, dass das Paradigma einer wettbewerbsorientierten kulturellen Leuchtturmpolitik nicht länger nur von neoliberalen Apologeten der Stadt- und Wirtschaftsentwicklung propagiert wird, sondern zunehmend auch von sich selbst als gesellschaftskritisch titulierenden Kulturschaffenden in alltäglichen Narrationen reproduziert wird. Ein Beispiel für eine derartige Universalisierung des Diskurses in der Stadtgesellschaft in Frankfurt ist ein Künstler, der sich selbst als gesellschaftskritisch beschreibt und sich für Künstlerateliers zu bezahlbaren Preisen in der Stadt einsetzt. Er sagt, wenn „beispielsweise die Stadt hierher gehen würde und sagen würde, wir kaufen jetzt mal irgendwie 500000 Quadratmeter von irgendjemandem [...] und vergeben die dann irgendwie zu 'nem superbilligen Preis an ganz viele Kreative, ich weiß nicht ob das gut wäre, ja? Weil, da gäb's eine wahnsinnige Schwemme an Kreativen [...] weiß nicht, ob wir denen nicht eine Konkurrenz produzieren, die die Stadt gar nicht verkraftet, ja, an Angebot von den Kreativen.“ (Interview mit dem Leiter des Künstlerhauses „Basis“ in Frankfurt vom 23. August 2011). Selbst er reproduziert eine marktorientierte Stadtpolitik, in dem nicht etwa kreative Vielfalt und eine reiche Kulturszene den Referenzrahmen für sein alltägliches Handeln bilden, sondern der Markt. Das Beispiel zeigt eindringlich, wie stark sich das Narrativ einer wettbewerbsorientierten kreativen Stadtentwicklung hegemonialisiert hat, so dass es sogar die alltäglichen Narrative sich als „kritisch“ bezeichnender Subjekte durchdringt.

Rancière (2008) bezeichnet diese Hegemonie als „postpolitischen Konsens". Dieser sei für eine bestimmte Form von Demokratie charakteristisch, wie sie sich seit dem Ende der konkurrierenden Ost-West-Ideologien durchgesetzt habe (Rancière, 2004:3f.). Sie kennzeichne sich durch eine „reduction of democratic life to the management of the local consequences of global economic necessity“ (ebd.: 4). Mit Hilfe von neuen konsensualen partizipativen Aushandlungsformen und Governance-Technologien würden Formen des Widerstandes und der Kritik derart in die verlängerten Arme des aktivierenden Staates eingehegt, dass Dissens de facto nicht mehr artikulierbar sei. „A post-democratic consensual policy arrangement has increasingly reduced politics to ,policing', to managerial consensual governing" (Swyngedouw, 2011:375). Diese „new forms of participatory governance" wie sie im Folgenden entlang des Fallbeispiels analysiert werden „,revolve around reflexive risk-calculation, accountancy rules and accountancy-based disciplining, quantification and bench-marking, articulated around a marketdriven hegemonic logic" (Oosterlynck und Swyngedouw, 2010:1580, für weitere kritische Ansätze zum Thema „Partizipation“ siehe auch Blakeley, 2010; Korf, 2009; Paddison, 2010; Rosol, 2011; Springer, 2011). 
„In short, research on urban and environmental change has been concerned primarily with policies/politics, but has been strangely silent about ,the political'. Put differently, research on changing regimes of urban and environmental governance has shown an overwhelming concern with the ontic level of urban and environmental policies“(Oosterlynck und Swyngedouw, 2010:1579).

Die folgende Analyse soll dieser konzeptionellen Forschungslücke begegnen und eine Alternative zur Mainstreamwissenschaft bieten, indem die hier als auf der ontischen Ebene operierend beschriebene, meist deskriptive Governance-Forschung theoretisch rückgebunden wird und damit neue Perspektiven radikaler Kritik neoliberaler Rationalitäten von Governance eröffnet werden.

\section{Theorien des Politischen und politischer Subjektivität}

Um eine konzeptionell geleitete Kritik am postpolitischen Konsens und hier konkret an neuen hegemonialen Subjektivierungsprozessen in konsensdemokratischen Partizipationsprozessen entwickeln zu können, wird im Folgenden auf Vertreter_innen neuer Theorien des Politischen zurückgegriffen, die in jüngster Zeit eine breite Rezeption erfahren (vgl. z.B. Bröckling und Feustel, 2010; Marchart, 2010; Rancière, 2004; Rancière, 2007) und auch vermehrt in der - vor allem anglophonen - Geographie rezipiert werden (vgl. z.B. Dikeç, 2005, 2007; Featherstone, 2008; Oosterlynck und Swyngedouw, 2010; Staeheli, 2008; Swyngedouw, 2005, 2011). Dazu wird zunächst die „Politische Differenz“ nach Marchart (2010) vorgestellt, die zwischen „,dem Politischen“als ontologischer und „der Politik“ als ontischer Kategorie unterscheidet. Jacques Rancière macht eine ähnliche Differenz auf. Er unterscheidet zwischen „Polizei“ als gegenwärtiger gesellschaftlicher Ordnung und „Politik“, die diese Ordnung aufbricht und verschiebt (Rancière, 2004, 2007; um Begriffsverwirrungen zu vermeiden, wird im Folgenden ausschließlich die Begriffsunterscheidung von Rancière - Politik/Polizei - verwendet, wenngleich sich inhaltlich auf unterschiedliche Theoretiker_innen der Theorien des Politischen bezogen wird). Im Rahmen dieses Artikels dienen die Unterscheidung zwischen Polizei und Politik (bgl. Kap. 3.1) sowie das daraus abgeleitete Konzept des „Unvernehmens“ (vgl. Kap. 3.2) als Folie, um den performativen Widerspruch zwischen dem Partizipationsverprechen städtischer Demokratie und neuen Ausschlüssen in der neoliberalen Stadt herausarbeiten und produktiv machen zu können (siehe Kap. 4.2).

\subsection{Polizei und Politik}

Unter Polizei versteht Jacques Rancière, was im Alltagsverstand als Politik bezeichnet wird, nämlich ,all the activi- ties which create order by distributing places, names, functions“" (Rancière, 1994:173 zit. n. Dikeç, 2005:175). Daher beschreibt er sie auch als eine spezifische Form der „Aufteilung des Sinnlichen“:

„Man nenne Aufteilung des Sinnlichen das allgemein inbegriffene Gesetz, das die Formen des Teilhabens bestimmt, in dem es zuerst die Wahrnehmungsweisen festlegt, in die sie sich einschreiben. [...] Eine Aufteilung des Sinnlichen ist die Art und Weise, nach der sich im Sinnlichen ein Verhältnis zwischen einem geteilten Gemeinsamen und die Einteilung exklusiver Anteile bestimmt. Diese Einteilung, die von ihrer sinnlichen Evidenz her die Einteilung der Anteile vorwegnimmt, setzt selber eine Aufteilung dessen, was sichtbar ist und was nicht, dessen was sich vernehmen lässt und was nicht, voraus" (Rancière, 2008:31).

Die polizeiliche Ordnung ist damit eine Form der Repräsentation, die bestimmt, was als Gemeinschaft angesehen wird (vgl. Dikeç, 2005:174). Als Aufteilung des Sinnlichen legt sie fest, was Gehör findet und was nicht; hier können ,,voices" in „noises"verwandelt werden. Polizeiliche Ordnung funktioniert als ein System der Regierung. ,, The police“ refers to an established social order of governance with everyone in their , proper' place in the seemingly natural order of things" (ebd.: 174).

Demgegenüber stellt Rancière die Politik als poststrukturalistische ontologische Differenzkategorie. Sie erscheint, wenn die polizeiliche Ordnung und die Aufteilung des Sinnlichen in Frage gestellt oder transzendiert werden. Politik ,calls into question the division of common and private, visible and invisible, audible and inaudible" (Rancière, 2004:6) und „existiert dort, wo die Rechnung/Zählung der Anteile und die Teile der Gesellschaft von der Einschreibung eines Anteils der Anteillosen gestört wird“ (Rancière, 2007:132). „Politics arises when the given order of things is questioned; when those whose voice is only recognized as noise by the police/policy order claim their right to speak, acquire speech, and produce the spatiality that permits exercising this right" (Swyngedouw, 2011:376).

\subsection{Das Unvernehmen und die Artikulation politischer Subjektivität}

Damit ist Politik eng mit der politischen Subjektkonstitution verbunden. ,[T]he only place one finds the unaccounted for is in the emergence of a political articulation, at a particular time and space, an emergence that becomes the claim of the unaccounted for to redefine the whole and to speak. [...] [T] he democratic theme precisely is not the inclusion of the excluded; it is the posture of the redefinition of the whole through the disruption of the police order by the institution of politics“ (Dikeç, 2005:177; Hervorheb. i.O.). Diese Form der Artikulation und politischen Subjektwerdung bezeichnet 
Rancière als „Unvernehmen“ (2007). „Die Fälle des Unvernehmens sind jene, bei denen der Streit darüber, was Sprechen heißt, die Rationalität der Sprechsituation selbst ausmacht." (ebd.: 10).

Judith Butler betont, dass der politische Prozess der Artikulation und Subjektwerdung ein performativer Akt ist. In Rekurs auf Derrida stellt sie heraus, dass ,die Kraft der Äußerung darin besteht, daß sie mit früheren, etablierten Kontexten bricht“" (Butler, 2008:221). Nach Butler werden in performativen Äußerungen und Akten nicht bloß soziale Konventionen bestätigt, vielmehr bestehe ,gerade darin, daß der herrschende, autorisierte Diskurs enteignet werden kann, eine Möglichkeit seiner subversiven Resignifikation“ (ebd.: 246, Hervorheb. i.O.). Erst in „,der Krise der Konvention, die das Aussprechen des Unsagbaren hervorruft", zeige sich nach Butler „die widerständige ,Wirkungskraft" des zensierten Sprechens, das im ,offiziellen' Diskurs auftaucht und der performativen Äußerung eine unvorhersehbare $\mathrm{Zu}$ kunft eröffnet.“ (ebd.: 223). Für sie ist das Enteignen performativer Äußerungen ein Moment des Politischen. „In der Politik kann Performativität gerade in dieser Form [der Enteignung, I.D.] gegen Herrschaft arbeiten. Jenes Moment, in dem ein Sprechakt ohne vorgängige Autorisierung dennoch im Vorgang seiner Äußerung Autorität gewinnt, kann einen veränderten Kontext seiner zukünftigen Rezeption antizipieren und setzen“ (ebd.: 250). „Die Logik der Iterierbarkeit“ zu erfassen, bedeutet damit für Butler, gesellschaftliche Widersprüche performativ (ebd. $142 \mathrm{ff}$.) werden zu lassen und damit „die Möglichkeit eines gesellschaftlichen Wandels zu begreifen" (ebd.: 230), weil sie genau auf der Ebene der performativen Äußerung des Subjekts Möglichkeiten des Widerstandes und der Veränderung hegemonialer Anrufungs-, Subjektwerdungs- und die Gesellschaft strukturierender Diskurse sieht.

Das iterative Moment politischer Subjektivierung bei Butler bzw. das Unvernehmen bei Rancière können damit als erster theoretischer Ausweg aus der fatalistischen Diagnose des im postpolitischen und neoliberalen Konsens zur Selbstführung und -verwertung verdammten Subjekts gelesen werden. Im Folgenden wird nun dem postpolitischen Konsens und seiner Herausforderungen durch neue Formen des Unvernehmens und politischer Subjektkonstitution anhand eines Fallbeispiels nachgegangen.

\section{KulturCampus Bockenheim}

Neue Formen der Artikulation von Unvernehmen und politischer Subjektivierung werden im Folgenden anhand der Fokussierung auf einen Teilaspekt des Planungskonflikts um den KulturCampus in Frankfurt herausgearbeitet. Die vorgestellte Fallstudie ist Teil eines DfG- Forschungsprojekts über die Entstehung und Ausdifferenzierung von Kreativpolitik als Prozess der Neuordnung des Städtischen im neoliberalen Zeitalter am Beispiel Frankfurts. Die Ergebnisse beruhen auf
23 qualitativen Interviews mit kreativ- und kulturpolitischen Akteuren, einer Korpusanalyse von Protokollen der Stadtverordnetenversammlung sowie lokaler Presseberichterstattung in der FR, FAZ und dem Journal Frankfurt, teilnehmender Beobachtung des Planungsprozesses sowie einer Analyse seiner Dokumentation. Diese Methodentriangulation ermöglicht einerseits hegemoniale städtische Handlungsrationalitäten zu rekonstruieren und andererseits ihre Subversion durch neue Formen der Artikulation und politischen Subjektivierung herauszuarbeiten.

Es ist der 16. Mai 2011, 19:30 Uhr in der Aula der GoetheUniversität in Frankfurt. Oberbürgermeisterin Petra Roth lädt zum zweiten Bürgerforum, um über die Zukunft des Campus Bockenheim zu sprechen. Der Hintergrund: Mit dem Wegzug der Goethe-Universität auf den Campus Westend wird eine 16,5 Hektar große Fläche frei, die unterschiedliche Phantasien von Politiker_innen, Stadtplaner_innen und Bürger_innen weckt, dieses große innenstadtnahe Areal aus einer Hand zu entwickeln. Heute wird das Konzept eines KulturCampus vorgestellt, ein Leuchtturm- und „bundesweites Vorzeigeprojekt" (DPA/Ihe, 2012) wie die Presse schreibt.

Was auf dem KulturCampus passieren soll, ist politisch umkämpft. „Oberbürgermeisterin Petra Roth“, betreibe dabei, wie das Journal Frankfurt schreibt, ihren „ganz eigenen Kampf“. „Partizipative Demokratie“ nennt sie das (Lorenz, 2011), um die Bürger_innen in einem partizipativen Planungsprozess zu „befrieden“ (Wygoda, 2012). Partizipation als Kampf? Konsensorientierte Planung als Waffe zur Herstellung eines Konsenses, der den Campus zu einem ,positiven Pendant zu Stuttgart 21“ (Michalzik, 2011) macht?

Auf dem Podium sind alle vertreten, die für den Planungsprozess wichtig sind. Der Geschäftsführer der ABG-Holding als zukünftige Eigentümerin des Areals, der Planungsdezernent der Stadt Frankfurt, ein Stadtplaner eines bedeutenden Frankfurter Planungsbüros, der Präsident der Hochschule für Musik und Darstellende Kunst als zukünftiger Pächter auf dem Gelände, der Kulturdezernent der Stadt sowie der Präsident der Goethe-Universität als scheinender Hausherr. Nur Bürgerinitiativen und politische Aktivist_innen fehlen. Ihnen war, trotz mehrfacher Nachfrage, die Teilnahme auf dem Podium verwehrt worden.

Der Ausschluss der Initiativen und Aktivist_innen ist verwunderlich. Laut Veranstalter_innen ist die „gleichberechtigte Kommunikation und Diskussion" (Freischlad + Holz/Herwarth + Holz/agl, 2011:10) eine zentrale Säule des partizipativen Planungsprozesses. Damit verorten sie sich explizit in einer Planungstradition, die sich auf die Habermas'sche Theorie kommunikativen Handelns (1981) stützt, nach der , in einem verständigungsrationalen Diskurs letztendlich gemeinsame Entscheidungen nicht per Machtanspruch der verschiedenen TeilnehmerInnen getroffen werden, sondern sich in einem intersubjektiven, kommunikativen Prozess die sog. ,Macht des besseren Arguments' durchsetzen kann" (Peters, 2008:314, vgl. auch Fainstein, 2000; Healey, 1997). Die Aktivist_innen und Initiativen hatten 
sich in den Wochen zuvor mit einer Vielzahl von Themen auseinandergesetzt, die auf dem Podium nicht vorgesehen sind: Sie hatten sich mit Möglichkeiten günstigen und genossenschaftlichen Wohnens auseinandergesetzt, Dokumentationen zur historischen Bedeutung von Gebäuden erstellt, über Bestands- und Denkmalschutz diskutiert und Konzepte für alternative Nutzungen erarbeitet, die ungehört bleiben. Politik begibt sich hier in einen ,,performativen Widerspruch" (Butler, 2008:142) zwischen Partizipationsversprechen und Ausschluss.

Am Abend des 16. Mai macht sich die Wut über die fehlende Möglichkeit der Beteiligung in der Aula der GoetheUniversität in einer ungewöhnlichen Weise Luft. Statt den Autoritäten auf dem Podium zuzuhören, erfreut sich an diesem Abend ein Spiel bei der Mehrheit der Anwesenden großer Beliebtheit, das von den Initiativen sowie aus dem Recht-auf-Stadt-Netzwerk Frankfurt entwickelt wurde und als Flugblatt auf allen Stühlen liegt: Es heißt Partizipationsbingo. Die Regeln des Spiels sind einfach: Wann immer einer, der in einer Tabelle auf dem Flugblatt vermerkten Begriffe einer neoliberalen kulturellen Stadtentwicklung durch jemanden auf dem Podium fällt, soll applaudiert werden. Es sind Begriffe wie „Leuchtturm“, „Kultur“ oder „Standort", die punkten. Der Gebrauch neoliberaler Wachstums-, Konkurrenz- und Unternehmensmetaphern in Broschüren und öffentlichen Auftritten zum KulturCampus war bereits zuvor von den Initiativen als Ausverkauf lokaler Interessen zugunsten der besseren Positionierung Frankfurts im internationalen Standortwettbewerb kritisiert worden (zur zentralen Rolle von Stadtimagepolitik für die neoliberale Stadtpolitik vgl. auch Mattissek, 2008; McCann, 2004, 2008). Wann immer fünf Begriffe in einer Reihe gefallen sind, soll aufgestanden und laut „Partizipation“ gerufen werden. Das Spiel nimmt einen unerwarteten Verlauf. Bereits die begrüßenden Worte der Oberbürgermeisterin ertrinken nicht nur in tosendem Applaus, sondern auch in euphorischem Jubel. Für die ersten Worte braucht sie eine Viertelstunde, weil sie immer wieder Worte benutzt, die auf den Flugblättern stehen und sofort johlender Applaus losbricht, der so laut ist, dass sie erst weitersprechen kann als dieser nach Minuten abebbt. Es dauert einige Zeit, bis das Podium versteht, was die von den Anwesenden spontan festgelegten Regeln des Abends sind. Nach einiger Zeit lässt Petra Roth von ihrem Statement ab und gibt das Mikrophon an das Publikum. Es entwickelt sich eine Auseinandersetzung über die Situation und über die Frage, wer wann im politischen Prozess das Recht zu sprechen hat. Diese Auseinandersetzung aber findet nicht nur inhaltlich-verbal zwischen den Beteiligten statt, sondern wird auch in der Form ihrer Inszenierung gespiegelt: Wann immer die Bürger_innen nun das Mikrophon in die Hand bekommen und Fragen des Rederechts ansprechen, sind sie von den im Publikum Sitzenden gut zu verstehen, nur das Podium kann sie nicht hören. Dies liegt daran, dass die Mikrophonanlage, die eigens für die Veranstaltung aufgebaut wurde, so arrangiert ist, dass alle Boxen auf das Publikum und weg vom Podium gerichtet sind. Ein Umstand, der ebenfalls zum Thema gemacht wird: „An der Anordnung der Boxen kann man sehen, wer an diesem Abend sprechen und wer zuhören soll. Das ist keine Teilhabe“ (Feldnotiz vom 16. Mai 2011). An der Weigerung des Publikums, in der von den Verantwortlichen vorgegebenen Form weiter zu diskutieren, kann Petra Roth durch das wutentbrannte Umdrehen der Boxen Richtung Podium ebenso wenig ändern, wie die mahnenden Worte des Planungsdezernenten, der betont: „Auch ich habe 68 hier Politik studiert, aber dort ist mit dem Kopf und nicht mit dem Kehlkopf diskutiert worden" (Feldnotiz vom 16. Mai 2011).

Zwei Aspekte des Bockenheimer Protests scheinen hier bemerkenswert, auf die im Folgenden nacheinander eingegangen wird: einmal die Art und Weise, wie der Planungsprozess im Vorfeld gerahmt und damit „Sachzwänge“ konstruiert wurden und zweitens, wie diese Festsetzungen über Inhalt und Form der möglichen Bürgerbeteiligung performativ herausgefordert wurden.

\subsection{Bürgerpartizipation als Prozessierung eines postpolitischen Konsenses}

Zentral für die Rahmung des Planungs- und Partizipationsprozesses war die Konstruktion der Wohnungsbaugesellschaft „ABG Holding“ - eine hundertprozentige Tochter der Stadt - als Unternehmen, das verpflichtet ist, gewinnorientiert zu wirtschaften. Die ABG hat das Areal des Campus im August 2011 vom Land Hessen gekauft. Durch den Verkauf der Fläche an die städtische ABG finanziert das Land den Umzug vom Campus Bockenheim auf den neuen Campus Westend und damit die Erneuerung der Goethe-Universität.

Die Konstruktion der ABG-Holding in öffentlichen Diskussionen und medialen Repräsentationen als Unternehmen sorgte dafür, dass die Debatten um die Nachnutzung des Bockenheimer Campus von Seiten der Stadt nie völlig ergebnisoffen geführt wurden. Vielmehr wurde auf diese Weise die Refinanzierung des Areals als ,ökonomische Rahmenbedingung" konstruiert, die den Beteiligten als unhinterfragbarer Sachzwang präsentiert wurde. Damit wurde der Planungsprozess wesentlich und entscheidend vorstrukturiert. Politische Gestaltungsspielräume, die die Stadt als Eigentümerin hat, wurden ausgeblendet. Dass es durchaus politische Gestaltungsspielräume gab und gibt, zeigt ein Blick in die Planungsgeschichte: Ursprünglich als Messeerweiterungsgelände geplant, sollten zunächst Bürogebäude für messe-, unternehmens- und finanznahe Dienstleistungen den Kauf des Areals lukrativ refinanzieren. Erst als im Rahmen der Finanzkrise 2008 die Nachfrage zur Refinanzierung nicht ausreichte, wurde die Idee des KulturCampus geboren. Eine von der Stadt durch die Ansiedlung von neun Kulturinstitutionen initiierte Entwicklung sollte das krisenbedingt fehlende private Investitionsinteresse kompensieren und Investitionen in anspruchsvolle Büros für Kreativschaffende und hochpreisiges Wohnen generieren. 
Weitere politische Handlungsspielräume aber waren durch die Art und Weise, wie die Pläne zum KulturCampus präsentiert wurden und wie an der Erarbeitung eines „Konsensplanes" partizipiert werden konnte, weitestgehend ausgeblendet. Eine solche „Vorformatierung“ im Vorfeld prozessiert einen postpolitischen Konsens, indem die Adressierung bestimmter Streitpunkte, die im Stadtteil schon seit längerem diskutiert wurden, durch die Struktur des Politik- und Partizipationsprozesses verhindert wird. Dies betrifft Eigentumsfragen, Fragen der Privatisierung weiter Teile des bislang öffentlichen Geländes, Probleme der Mietsteigerung und Verdrängung durch die Aufwertung des Stadtteils Bockenheim zur „Innenstadtlage II“ im Mietspiegel 2011, die drohende Gentrifizierung durch Aufwertungsprozesse auf dem KulturCampus, die Abrissplanung denkmalgeschützter und historisch bedeutsamer Nachkriegsarchitektur sowie Fragen gewachsener Stadtteilkultur auf dem Campus. Und auch am Abend des 16. Mai 2011 können die zentralen Forderungen nach einem höheren Wohnanteil auf dem Campus, dem Erhalt der Kindertagesstätte, historisch bedeutsamer Gebäude und gewachsener sozio-kultureller Strukturen auf dem Campus nicht adressiert werden. Subjektpositionen, die Kritik jenseits des gesetzten Planungsrahmens artikulieren wollen, sind im Planungsprozess schlicht nicht vorgesehen. Anliegen, die nicht kulturalisiert oder in den Dienst einer kulturinduzierten Stadtentwicklung gestellt werden können, werden schlicht nicht gehört (Ronneberger, 2012:61). Forderungen nach einer sozialen Gestaltung des Campus werden als „Utopien“ tituliert und als „unrealistisch“ oder ,nicht bezahlbar"ausgeschlossen (Aussagen von Brigitte Holz von Freischlad + Holz, Moderatorin der Planungswerkstätten zum KulturCampus Bockenheim, Feldnotiz vom 17. Februar 2012). Fragen - wie sich im weiteren Planungsprozess noch häufiger zeigen soll -, die das Paradigma einer wachstumsorientierten Stadtentwicklung in Frage stellen, sind im Planungsprozess selbst nicht mehr verhandelbar.

Genau auf diese Weise führt Konsensdemokratie „zu einer Stigmatisierung der sozialen Bewegungen, die gegen eine Gleichsetzung von Demokratie mit der öffentlichen Verwaltung ökonomischer Notwendigkeiten kämpfen. Die vorgebliche Rückkehr der Politik" durch den Prozess der Bürgerbeteiligung ist, so Rancière, ,in Wirklichkeit ihre Auflösung“" (Rancière, 2004:4; Übersetzung I.D.).

„Die Gleichsetzung von Demokratie mit Konsens ist die gegenwärtige Form ihres Verschwindens. Konsens meint nicht einfach das Auslöschen von Konflikten zum Wohle gemeinschaftlicher Interessen. Konsens meint das Auslöschen der umkämpften und konflikthaften Natur dessen, was wir als Gemeinschaft bezeichnen. [...] Mit anderen Worten, stützt sich der konsensuelle Staat auf eine globale ökonomische Notwendigkeit, die als Sachzwang präsentiert wird, um Konflikte über das, was Gemeinschaft ist, in ihre internen Probleme zu transformieren“ (ebd.: 7).

In Anlehnung und Weiterentwicklung von Gramscis berühmtem Satz Hegemonie ,zeichnet sich durch die Kombination von Zwang und Konsens aus“ (1991ff.:1610), könnte man hier von „Konsens gepanzert mit konstruiertem Sachzwang“ sprechen. In der neoliberalen Stadt wird das Versprechen, jeder könne seine Stimme in den politischen Prozess einbringen, zunehmend nicht mehr eingelöst. Diese Tatsache aber ist im Modus partizipativer Demokratie selbst nicht adressierbar, weil diese sich durch das Gleichheits- und Partizipationsversprechen erst konstituiert.

\subsection{Artikulation von Unvernehmen - oder wie man mit dem Kehlkopf diskutiert}

Was aber genau ist am 16. Mai 2011 passiert? Durch das Bingo wird der Widerspruch partizipativer Demokratie zwischen Partizipationsversprechen und Ausschluss von als ökonomischen Sachzwängen gerahmten Fragen performativ. Hier werden solche Formen des Ausschlusses verhandelbar, die unter dem Gleichheits- und Partizipationspostulat als polizeiliche Ordnung der gegenwärtig hegemonialen Demokratieformation nicht artikulierbar sind. Durch die Thematisierung des performativen Widerspruchs gelingt es, die postpolitischen Regeln des Spiels selbst zum Thema zu machen, zu diskutieren und zu kritisieren. Im Gegensatz zu vielen anderen Formen des Widerstandes gegen stadtentwicklungspolitische Maßnahmen ist es hier gelungen, den postpolitischen Konsens einer renditeorientierten, kulturinduzierten Stadtpolitik wieder in einen politischen Prozess zu überführen.

Der Prozess der Artikulation im Partizipationsbingo kann als „Unvernehmen“ (vgl. Rancière, 2007:132) bezeichnet werden, weil hier ,der Streit darüber, was Sprechen heißt, die Rationalität der Sprechsituation selbst ausmacht" (ebd.: 10). Genau an diesem Punkt ging der politische Prozess über die Aufführung der polizeilichen Ordnung im Rancière'schen Sinne hinaus und zeigte sich als das, was Slavoj Žižek als „eigentlichen politischen Akt" bezeichnet:

„Der eigentliche politische Akt (die Intervention) ist nicht einfach etwas, was innerhalb der existierenden Verhältnisse gut funktioniert, sondern etwas, was gerade den Rahmen verändert, der festlegt, wie die Dinge funktionieren. [..., Sie] ist die Kunst des Unmöglichen: Sie verändert gerade die Parameter dessen, was in der existierenden Konstellation als ,möglich“ betrachtet wird“ (Žižek, 2010:273, Hervorheb. i.O.).

Es konnte eine Verschiebung der „Aufteilung des Sinnlichen" (Rancière, 2008:31) dahingehend festgestellt werden, dass auf einmal politische Subjekte gehört wurden, deren Protest - auch und ganz besonders im Rahmen des Partizipationsbingos - nur als „Lärm“ zu vernehmen waren. Es sei an 
dieser Stelle noch einmal auf den Planungsdezernenten verwiesen, der durch seinen Kommentar, früher habe man mit dem Kopf und nicht mit dem Kehlkopf diskutiert, den Aktivist_innen ihre Stimmen abgesprochen hat.

„Es ist schon toll, was diese PerformanceKünstler machen. So etwas hätten wir als Bürgerinitiative nie gekonnt. Im Anschluss an diese Veranstaltung [das Bürgerforum vom 16. Mai 2011, I.D.] wurden ich und Tim Schuster vom offenen Haus der Kulturen von der FR zu einem Dialoggespräch mit Petra Roth und Frank Junker [Geschäftsführer der ABG-Holding, I.D.] eingeladen. Das hätte es sonst nie gegeben. Das war das erste und einzige Mal im gesamten Prozess, wo ein Treffen auf Augenhöhe möglich war." (Interview mit der Sprecherin der ,Initiative Zukunft Bockenheim“ in Frankfurt vom 28. September 2011)

Dies war Ausgangspunkt für weitere Aktionen und Forderungen, die zur Folge hatten, dass Streitpunkte, die bis dato im Planungsprozess nicht adressierbar waren, nicht nur adressiert, sondern politisch auch verändert werden konnten. Dies sind im Einzelnen der Erhalt eines Teils der historischen Gebäude, darunter der Erhalt des Studierendenhauses als offenes Haus der Kulturen, der Erhalt der Kindertagesstätte sowie eine Erhöhung des Wohnanteils auf 40 Prozent.

\section{Wege aus der Krise des kreativen Subjekts}

Ausgelöst durch das Partizipationsbingo artikulieren Subjekte Positionen, die bis dato im Diskurs und politischen Prozess nicht möglich waren. Der Prozess der Artikulation geht notwendigerweise mit der Überwindung althergebrachter Diskurse und Subjektpositionen einher. Hier zeigt sich das politische Moment der Subjekte. Politische Interventionen ,disrupt identities and discourses [...] In short, it is the ,failure“ of the structure, and as we have seen of those subject positions which are part of such a structure, that ,compels' the subject to act, to assert a new its subjectivity" (Howarth und Stavrakakis, 2000:13). Politische Identitäten werden folglich durch das Aufbrechen bestehender Diskurse geformt. Im Fallbeispiel hat sich dies dadurch gezeigt, dass die Regeln der Aushandlung im Partizipationsprozess verschoben und damit vorher nicht verhandelbare Forderungen (Erhöhung des Wohnanteils und Erhalt des Studierendenhauses) politisch verhandelbar wurden. Damit zeigt sich, dass die Krise des kreativen Subjekts nur dann überwunden werden kann, wenn es ihm gelingt, die eigene ihm zugewiesene Subjektposition in einem Akt des Unvernehmens und im Rahmen der Artikulation politischer Subjektivität zu subvertieren und damit zu transzendieren.

„Die Politik existiert, sofern singuläre Formen der Subjektivierung die Formen der ersten Einschreibung der Identität zwischen dem Ganzen der
Gemeinschaft und dem Nichts, das sie von sich selbst, d.h. von der einfachen Zählung der Teile trennt, erneuern. Die Politik hört auf zu sein, wo dieser Abstand keinen Ort mehr hat, wo das Ganze der Gemeinschaft restlos in die Summe seiner Teile aufgeht“" (Rancière, 2007:132).

Das Beispiel zeigt sehr eindringlich, dass konsensuale und hegemoniale Rationalitäten der Regierung von Stadt zwar machtvoll, aber keine Sachzwänge sind, sondern politisch umkämpft. Es zeigt auch, dass Widerstand sich nicht einfach damit begnügen kann, Forderungen zu stellen, weil diese in postpolitischen Aushandlungsformen immer wieder eingehegt werden können, sondern dass Protest performativ in die Regeln des Spiels intervenieren muss, wenn er etwas verändern will. Nur so zeigt sich ,das performative Moment der Kritik“, das ,sich mit in konkreten Kontexten stattfindendem Handeln befasst, in welchem das Subjekt seine Umwelt und die Umwelt ihr Subjekt mit offenem Ausgang verändert“ (Nestler, 2011:95).

\subsection{Politische Subjektivität als eine Politik des Ortes und Recht auf Stadt}

In den Theorien des Politischen ist die Artikulation von Unvernehmen und politischer Subjektwerdung immer eine Politik des Ortes. „Raum ist das kontingente Ergebnis einer artikulatorischen Praxis, die überhaupt erst die Positionen zu einer Topographie verknüpft. Diese Praxis ist nichts anderes als Politik, eine Praxis nämlich [...] der Verräumlichung" (Marchart, 2002). Daher ist es von zentraler Bedeutung, ,daß die konfliktuelle Verfaßtheit der Gesellschaft, der Politik und letztlich des öffentlichen Raumes nicht verdrängt oder verkleistert wird, wie das in Konsensmodellen der Fall ist" (ebd.). Vertreter_innen der neuen Theorien des Politischen weisen auf den konfliktiven Charakter radikaler Demokratie hin, der sich immer nur an konkreten Orten artikulieren kann (ebd., vgl. z.B. auch Rancière, 2007:148; Springer, 2011). Umso wichtiger ist es, Orte zu schaffen und zu erhalten, an denen sich diese Antagonismen artikulieren können, wie es derzeit von der Recht-auf-Stadt-Bewegung gefordert wird. „Öffentlichkeit ist also kein Konsensraum, sondern ein Dissensraum. Der urbane öffentliche Raum wird [...] durch Konflikt erzeugt, nicht durch einen Konsens, der auf rationale und prozedurale Metaregeln zurückgreifen könnte“ (Marchart, 2002).

\subsection{Performative Wissensproduktion und Intervention in den Stadtraum}

Das Partizipationsbingo hat an einem ganz bestimmten Punkt des Konflikts interveniert und zur Anerkennung ganz bestimmter Stimmen geführt. Das Ereignis war wichtig, aber singulär. Das Bingo hat im Endeffekt nicht zu signifikanten Änderungen der konsensualen Planung in Bockenheim 
geführt, von einer ernsthaften Infragestellung des postpolitischen Konsenses in der unternehmerischen Kreativstadt ganz zu schweigen. Und dennoch hat dieser Artikel allen Grund, sich diesem Ausdruck zivilen Ungehorsams zu widmen. Die Analyse des Partizipationsbingos als Ereignis des Unvernehmens und Artikulation politischer Subjektivität stellt selbst einen performativen Akt der Wissensproduktion dar. Es konnte herausgearbeitet werden, dass sich in dieser Form des Unvernehmens eine neue Qualität des Widerstandes gegen postpolitische und neoliberale Formen der Stadtentwicklung und Subjektivierung zeigt. Sie ist in der Lage, die Regeln des postpolitischen Spiels der partizipativen Planung selbst zum Gegenstand und damit verhandelbar zu machen. Indem Forschung die Qualität solch neuer performativer Interventionsformen herausarbeitet und in andere Öffentlichkeiten trägt, wird es möglich, sie performativ weiter zu schreiben und in anderen städtischen Kontexten zu erproben. Solche Formen der Intervention in Wissensproduktion sind wichtig, wenn kritische Wissenschaft ihre Aufgabe machtanalytisch verändernd - und nicht machtanalytisch reifizierend - wirken zu wollen, ernst nimmt.

In der Recht-auf-Stadt-Bewegung oder den performance studies (z.B. Deck und Sieburg, 2011; Hartmann et al., 2012; Schmidt-Wulffen, 2008) werden derzeit interessante städtische Interventionsmöglichkeiten erforscht, bestehende Aufteilungen des Sinnlichen in Frage gestellt und neue Artikulationsformen politischer Subjektivität erprobt. Diese neuen Formen der Ästhetisierung und Politisierung von Subjektivität stellen eine Chance dar, den postpolitischen Konsens gegenwärtiger Stadtentwicklung in Frage zu stellen sowie neue Formen von Unvernehmen und politischer Subjektwerdung als konkrete Formen der Interventionen in den Stadtraum, als Politik des Ortes im Sinne eines Rechts auf Stadt, zu erkunden und praktisch werden $\mathrm{zu}$ lassen. Inwiefern es gelingen wird, solchen neuen Interventionsformen eine breitere gesellschaftliche Bühne zu geben, ist offen. Erkenntnisse und vor allem Praktiken aus dem Bereich der performance studies und ihrer interventionistischen Praxis können die geographische Diskussion um politische Subjektivität und ihre Formen der urbanen Raumproduktionen - gerade auch im Kontext der Rechtauf-Stadt-Debatte - deutlich bereichern. Sie eröffnen dem Kreativsubjekt neue Möglichkeiten der Auseinandersetzung mit seiner Krisenhaftigkeit und Repolitisierung.

Edited by: O. Söderström

Reviewed by: two anonymous referees

\section{Literatur}

Blakeley, G.: Governing Ourselves: Citizen Participation and Governance in Barcelona and Manchester, IJURR, 34, 130-145, 2010.

Boltanski, L. und Chiapello, È.: Der neue Geist des Kapitalismus, Konstanz, 2006.
Bröckling, U. und Feustel, R. (Hrsg.): Das Politische denken. Zeitgenössische Positionen, Bielefeld, 2010.

Butler, J.: Haß spricht. Zur Politik des Performativen, Frankfurt am Main, 2008.

Deck, J. und Sieburg, A.: Politisch Theater machen. Neue Artikulationsformen des Politischen in den darstellenden Künsten, Bielefeld, 2011.

Dikeç, M.: Space, politics, and the political, Environ. Plann. D, 23, 171-188, 2005.

Dikeç, M.: Badlands of the republic. Space politics and urban policy, Malden MA u.a., 2007.

DPA/Ihe: Kulturcampus soll bundesweites Vorzeigeprojekt werden, in: Bild Zeitung Frankfurt regional, 28. März 2012.

Ehrenberg, A.: Das erschöpfte Selbst. Depression und Gesellschaft in der Gegenwart, Frankfurt am Main, 2008.

Fainstein, S. S.: New Directions in Planning Theory, Urban Aff. Rev., 35, 451-478, 2000.

Featherstone, D.: Resistance, space and political identities. The making of counter-global networks, Malden Mass u.a., 2008.

Freischlad + Holz/Herwarth + Holz/agl: Dialogischer Planungsprozess Kulturcampus Frankfurt, Dokumentation Auftaktforum, Protokoll 1 im Auftrag der ABG Frankfurt Holding sowie der Stadt Frankfurt, Frankfurt am Main, 2011.

Gibson, K.: Regional subjection and becoming, Environ. Plann. D, 19, 639-667, 2001.

Gramsci, A.: Gefängnishefte, 1-10, Hamburg, 1991ff.

Gregson, N. und Rose, G.: Taking Butler elsewhere. performativities, spatialities and subjectivities, Environ. Plann. D, 18, 433$452,2000$.

Habermas, J.: Theorie des kommunikativen Handelns, Frankfurt am Main, 1981.

Hartmann, D., Lemke, I., und Nitsche, J.: Interventionen. Grenzüberschreitungen in Ästethik, Politik und Ökonomie, Paderborn, 2012.

Healey, P.: Collaborative planning, Shaping places in fragmented societies, Vancouver, 1997.

Howarth, D. und Stavrakakis, Y.: Introducing discourse theory and political analysis, in: Discourse theory and political analysis. Identities, hegemonies and social change, Herausgeber: Howarth, D., Manchester, 1-24, 2000.

Korf, B.: Partizipation als Ausnahmezustand, Geogr. Helv., 64, 98 105, doi:10.5194/gh-64-98-2009, 2009.

Loacker, B.: Kreativ Prekär. Künstlerische Arbeit und Subjektivität im Postfordismus, Bielefeld, 2010.

Lorenz, J.: Kultur-Campus. Was Bürger wollen, Journal Frankfurt, 22. Februar 2011.

Manske, A.: Prekarisierung auf hohem Niveau oder: Risikolage Kreativarbeit, in: Kreativen: Wirkung. Urbane Kultur, Wissensökonomie und Stadtpolitik, Herausgeber: Heinrich-BöllStiftung, Berlin, 48-52, 2008.

Marchart, O.: Kunst, Raum und Öffentlichkeit(en). Einige grundsätzliche Anmerkungen zum schwierigen Verhältnis von Public Art, Urbanismus und politischer Theorie, in: transversal - eipcp multilingual webjournal, Nr. 01, online unter: http://eipcp.net/ transversal/0102/marchart/de, zuletzt abgerufen: 29. April 2012, 2002.

Marchart, O.: Die politische Differenz. Zum Denken des Politischen bei Nancy, Lefort, Badiou, Laclau und Agamben, Berlin, 2010. 
Mattissek, A.: Die neoliberale Stadt. Diskursive Repräsentationen im Stadtmarketing deutscher Großstädte, Bielefeld, 2008.

McCann, E.: "Best places": interurban competition, quality of life and popular media discourse, Urban Stud., 41, 1909-1929, 2004.

McCann, E. J.: Livable City/Unequal City: The Politics of PolicyMaking in a "Creative" Boomtown, Revue Interventions économiques, 37, online unter: http://interventionseconomiques. revues.org/489, zuletzt abgerufen: 29. April 2012, 2008.

McDowell, L.: Thinking through work: complex inequalities, constructions of difference and trans-national migrants, Prog. Hum. Geog., 32, 491-507, 2008.

McRobbie, A.: Reflections on Precarious Work in the Cultural Sector. in: Governance der Kreativwirtschaft, Diagnosen und Handlungsoptionen, Herausgeber: Lange, B., Kalandides, A., Stöber, B., und Wellmann, I., Bielefeld, 123-138, 2009.

Menke, C. und Rebentisch, J.: Kreation und Depression. Freiheit im gegenwärtigen Kapitalismus, Berlin, 2010.

Michalzik, P.: Man nennt es Zukunft, Frankfurter Rundschau, 11. April 2011.

Nestler, S.: Performative Kritik. Eine philosophische Intervention in den Begriffsapparat der Cultural Studies, Bielefeld, 2011.

Oosterlynck, S. und Swyngedouw, E.: Noise reduction: the postpolitical quandary of night flights at Brussels airport, Environ. Plann. A, 42, 1577-1594, 2010.

Paddison, R.: Protest in the Park. Preliminary Thoughts on the Silencing of Democratic Protest in the Neoliberal Age, Variant, 39/40, 20-28, 2010.

Peck, J.: Struggling with the Creative Class, IJURR, 29, 740-770, 2005.

Peck, J.: Recreative City: Amsterdam, Vehicular Ideas and the Adaptive Spaces of Creativity Policy, IJURR, 36, 462-485, 2012.

Peters, D.: PlanerInnen als ,deliberative practitioners“ - Auf dem Weg zu einem neuen, diskursiven Pragmatismus in der Planung(stheorie)? in: Strategieorientierte Planung im kooperativen Staat, Herausgeber: Hamedinger, A., Wiesbaden, 309-321, 2008.

Pongratz, H. J. und Voß, G. G.: From employee to "entreployee": Towards a "self-entrepreneurial" work force?, Concepts and Transformation, 8, 239-254, 2003.

Rancière, J.: Introducing disagreement, Angelaki, 9, 3-9, 2004.

Rancière, J.: Das Unvernehmen. Politik und Philosophie, Frankfurt am Main, 2007.

Rancière, J.: Zehn Thesen zur Politik, Zürich u.a., 2008.

Reckwitz, A.: Die Erfindung des Kreativsubjekts. Zur kulturellen Konstruktion von Kreativität, in: Unscharfe Grenzen. Perspektiven der Kultursoziologie, Herausgeber: Reckwitz, A., Bielefeld, 235-258, 2008.
Ronneberger, K.: „Diese Stadt haben wir satt. Wie sie ist, ist sie Mist“. Ein Rückblick zum Global City-Aufstieg Frankfurts, in: Wem gehört Frankfurt? Dokumentation des aktionistischen Kongresses vom März 2012, organisiert aus dem „Wem gehört die Stadt?"-Netzwerk = Forum Humangeographie, Herausgeber: AK Kritische Geographie, Frankfurt a.M., 9, 54-64, 2012.

Rosol, M.: Governing cities through community participation. Zur Deutung und Kritik städtischen Regierens über Partizipation, Habilitationsschrift, Goethe-Universität Frankfurt am Main, 2011.

Schipper, S.: Genealogie und Gegenwart der ,unternehmerischen Stadt". Neoliberales Regieren in Frankfurt am Main zwischen 1960 und 2010, Unveröffentlichte Dissertation, Frankfurt am Main, 2012.

Schmidt-Wulffen, S.: The artist as public intellectual?, in: Schriften der Akademie der Bildenden Künste Wien, Wien, 1, 2008.

Springer, S.: Public Space as Emancipation: Meditations on Anarchism, Radical Democracy, Neoliberalism and Violence, Antipode, 43, 525-562, 2011.

Staeheli, L. A.: Political geography: difference, recognition, and the contested terrains of political claims-making, Prog. Hum. Geog., 32, 561-570, 2008.

Strüver, A.: Der Konstruktivismus lernt laufen: „Doing morethan-representational geography“, Soc. Geogr., 6, 1-13, doi:10.5194/sg-6-1-2011, 2011a.

Strüver, A.: Das Private ist politisch - "again": Die Politik der Gesundheitsprävention als performative Biopolitik, in: Die Politik räumlicher Repräsentationen. Beispiele aus der empirischen Forschung = Forum Politische Geographie, Herausgeber: Dzudzek, I., Reuber, P., und Strüver, A., Münster, 6, 315-339, 2011 b.

Swyngedouw, E.: Governance innovation and the citizen: The Janus face of governance-beyond-the-state, Urban Stud., 42, 19912006, 2005.

Swyngedouw, E.: Interrogating post-democratization: Reclaiming egalitarian political spaces, Polit. Geogr., 30, 370-380, 2011.

Valentine, G.: Theorizing and Researching Intersectionality: A Challenge for Feminist Geography, RTPG, 59, 10-21, 2007.

von Osten, M. und von Bismarck, B. (Hrsg.): Norm der Abweichung, Zürich, 2003.

Wygoda, H.: Kein Konsens über Campus. Bürger sehen noch offene Fragen, doch die Stadt stellt schon Pläne vor, Frankfurter Neue Presse, 29. März 2012.

Žižek, S.: Die Tücke des Subjekts, Berlin, 2010. 